\title{
Examining the Use of Information Systems to Preserve Indigenous Knowledge in Uganda: A Case from Muni University
}

\author{
Josephat O. Oroma \\ Muni University/Department of Computer and Information Science, Arua, 256, Uganda \\ Email: j.oling@muni.ac.ug \\ Guma Ali \\ Muni University/Department of Computer and Information Science, Arua, 256, Uganda \\ Email: a.guma@muni.ac.ug
}

Received: 12 February 2018; Accepted: 08 March 2018; Published: 08 May 2018

\begin{abstract}
Indigenous Knowledge (IK), can be preserved using Information Systems in order to protect cultural heritage and disseminate local knowledge for development. This knowledge often passed on orally for generations has become significant in searching for answers to several world's critical problems, are at risk of becoming extinct. This "traditional wisdom" is highly useful in solving complex problems of health, agriculture, education, use of natural resources and the environment. The main challenges of IK are inadequate documentation and diminishing transmission channels. Both descriptive and quantitative methods are used in this study that focuses on highlighting the importance of indigenous knowledge in the sustainable development process and illustrating ways in which technology can be used to preserve it, thereby enriching the development process from a holistic perspective. This research strengthens the preservation of local IK, enhances its adoption in the formal educational settings, leads to improvement in scientific knowledge development and inspire sustainable community development using a holistic approach.
\end{abstract}

Index Terms-Information Systems, Indigenous Knowledge, ICT.

\section{INTRODUCTION}

Indigenous Knowledge (IK) is the local knowledge that is embedded in the unique cultural practices of regions or local communities and is often orally passed on from generation to generation by elderly knowledgeable people [1]. Similarly, [2] commented that Indigenous Knowledge can be comprehensively and conclusively defined as:

The knowledge that people in a given community developed over time, and continue to develop. It is based on experience; often tested over centuries of use; adapted to local culture and environment and is dynamic and changing. It is not confined to rural people. Rather, any community possesses the indigenous knowledge, rural or urban, settled or nomadic, original inhabitants and migrants. IK is more than just technologies and practices. It includes information; practices; technologies; beliefs; tools; materials; experimentation; biological resources; human resources; education and communication.

Often referred to as "traditional wisdom", IK is accumulated through long-term interaction with the natural environment and includes knowledge about traditional technologies like tools and techniques for hunting, agriculture and food security, fishing, midwifery, education, health-care, ecological knowledge, and conservation and became essential for the existence and survival of local inhabitants [3], and constitutes skills for existence. [4] summarized the nature of Indigenous Knowledge into - (a) locally bound, indigenous to specific area (b) culture and context specific (c) nonformal knowledge (d) orally transmitted and generally not documented (e) dynamic and adaptive and (f) closely related to survival and subsistence for many people worldwide.

IK is increasingly becoming essential as the world consolidates globalization and the key to the development of local communities lie in IK. The concept of globalization increases the value of local information [5], especially when meaningfully stored and accessed from anywhere on demand. However, this essential local wisdom has been ignored by Western partners particularly former colonialists, in the development strategy for emerging regions and forgotten in the globalization of modern science and technology [5]. The oral tradition used by local communities to preserve IK is no longer effective in this generation.

The younger generation, often known as the "dot com" generation are important stakeholders in the development process but are ignorant about IK, are also salient users of technology and digitizing IK will provide to them adequate access to IK through technology. As [6] 
explains, the older African generations are conversant with IK, but the "net generations" largely ignore such knowledge. Using Information Systems revitalizes endangered cultures, improves economic independence and sustainability while increasing community-based involvement in planning and development [7].

IK is a solution to problems of people living out of access to modern development because it represents a heritage and a rich body of time-tested local knowledge. [8] indicates that IK provides problem-solving strategies for locals in poor settings and is an integral part of development process because the key to sustainable development is knowledge and not capital. There are local experts who deal with local problems. For example, in some tribes, there are traditional healers to treat whoever falls sick. These healers have enormous accumulated knowledge of herbs used for treating several types of ailments. Such knowledge is therefore passed on from one generation to the next by observation, narration and practice. There are also rainmakers and stoppers to act in situations where climate has changed.

IK has a commercial value as revealed in [9], that IK has the potential of being translated into commercial benefits by providing leads for the development of useful practices and processes for the benefits of mankind. Previous studies [10] report that IK is an important resource in the development process and sharing IK within and across communities can enhance crosscultural understanding and promote the cultural dimension of development. Also [11] states that IK provides the basis for local-level decision-making about many fundamental aspects of day-to-day life. Several communities have used their local knowledge to make decisions that affects their localities such as food security, health and resource sharing.

The objectives of the study are to a) identify the relevance of Indigenous Knowledge towards sustainable community and national development, b) investigate the challenges Indigenous Knowledge face in this era of globalization and advancement of technology, c) examine how to use Information Systems to preserve and disseminate Indigenous Knowledge in this context.

The biggest challenge of IK is inadequate documentation, lack of preservation strategies and poor dissemination [5]. It is common knowledge that the oral tradition excludes documentation, but relies heavily on transmitting information from one generation to the next by word of mouth. Leading to risks of IK becoming extinct if not preserved using modern methods. Again, oral traditions have become largely unavailable or inaccessible to many young people, who no longer have access to non-formal learning, many have decreased fluency in their mother tongues to keep them communicating to elders [12]. Also, formal education programs only promote foreign values, science, and health programs and downplay the importance of IK rendering it inaccessible. Other studies [13], and [14] note that IK is under threat from modern technology because even in remote areas the powers that push global or just non-local content such as radio and television broadcasting and advertising among others, are much stronger than those pulling local content. Kelly [15] identified political pressures, cultural integrity, social and economic pressures, territorial pressures, exploitation of traditional knowledge, development policy, and globalization and trade liberalization as threats to Indigenous traditional knowledge. Also [16] states that that Indigenous Knowledge has largely been marginalized, neglected and suppressed due to ignorance and arrogance, politics, and the dominant ideology of a particular historical period. He further added that Indigenous Knowledge is threatened by socialization, the education system, the influence of western technology. Furthermore [17] argue that "poor attitudes, knowledge culture and personal characteristics (age, gender, status, wealth, political influence and so on) also affect perceptions, actions, and access to knowledge in the local communities" (p. 176). According to [18], colonial and postcolonial education, which has excluded IK are factors affecting the transmission of IK, and the deliberate or inadvertent destruction of local knowledge [19], [20]. Other studies [21] also noted that younger generations that are exposed to Western education are less interested in indigenous knowledge, viewing such knowledge as outdated and primitive.

\section{RELATED WORKS}

It is clearly evident that few studies have been done on IK preservation especially in the African context as reflected in the significantly low number of published articles related to this topic. Many of the available articles are publications of studies about IK in Asia and South American countries. This study reviews available literature related to IK and technology under the following sub-headings; technological advancement, IK systems, ICT for IK preservation and a theoretical framework.

\section{A. Technological Advancement}

With the rapidly emerging new technology, digitization and dissemination of knowledge has created what is known as the knowledge economy whereby those in need of information and knowledge can easily access it with a click, from anywhere at any time. Technology has changed the way we conduct business, communicate to each other, and relate to each other. While technology is becoming social, in other ways it has isolated people and has reduced the time people gather to chat and exchange ideas. Technology and ICTs in general and Information Systems, in particular, has the potential to aid the identification, collection, storage, and dissemination of IK in order to bridge the IK gap. For example, with the coming of Television, Internet, and broadcast radios, the time community and family members come together to tell stories, or engage in activities that transmit IK from one person to another has greatly reduced since many members especially the young ones prefer to sit glued to their gadgets to watch, listen to their favorite program being aired. These kinds of technology have changed 
human behavior in a way that it affects the traditional indigenous systems, which is broadly based on oral tradition. This is the reason to utilize this kind of media to enhance the preservation of IK because it is on the verge of becoming extinct [16]. For oral tradition to flourish, people to people interactions must be constant and frequent. In this $21^{\text {st }}$ century, technology has disrupted such interactions greatly thereby interfering with IK transmission.

\section{B. Indigenous Knowledge System}

The traditional knowledge has its own system and method of preservation. It is often passed on from one generation to the next using oral tradition. This is known as the Indigenous Knowledge System (IKS). The elders are central to this system because they have lived longest, meaning that they have experienced more and therefore have more knowledge as compared to the rest of the community members. These elders are always looked up to, in case of problems or in situations of new problems without solutions these elders would gather and brainstorm to find solutions mainly basing on knowledge transfer from previous experience with other problems where solutions worked successfully. There were specific channels for knowledge distribution. For example, storytelling was one way to transmit knowledge, songs and traditional dances were also used as means to transmit knowledge. The others included ceremonies performed during events such as harvesting, childbirth and child naming, and other cultural rites like circumcision which are often performed to initiate boys into manhood. An earlier study [10] suggests that local knowledge systems often combine specific and the general aspect of knowledge with great ease since everybody has local everyday knowledge about the environment. They further explained that IK systems are geared towards dealing with diversity in both natural environment and social organization and continues to evolve over time and they differ from scientific knowledge in their capacity to deal with local problems. Other reports [22] indicates that, in IKS, generation of knowledge starts with "stories" as the base units of knowledge; proceeds to "knowledge," an integration of the values and processes described in the stories; and culminates in "wisdom," an experiential distillation of knowledge. IKS is holistic and people centered in the sense that it strives to resolve human problems using time-tested solutions and ecological in nature as stated in [10], IKS are typically human-centered, very diverse, applying technology of local origin with strong cross-linkages and is developed after the years of experiences and experiments, trial and error and incremental refinement. Again [23], suggests that in order for proper understanding and incorporating of IKS for sustainable socio-economic development and poverty alleviation, the scientific community perhaps need to "unlearn" our old view of knowledge and should grasp the importance of local or indigenous peoples view.

The traditional or IKS is challenged by the significant number of factors affecting its documentation and dissemination. And [24] explains that information flow in an oral context is controlled by attitudes, perceptions, norms, values and belief systems inherent to indigenous people. He even cited an example that, when people experience an information need, they will approach a knowledgeable person whom they trust. Other factors affecting IKS include things like formal education introduced by the colonialists and western religion such as Christianity as well as Islam. Furthermore [19] revealed that IKS have suffered for several decades from several strategies of disinformation embedded in westerncentric, colonial and post-colonial education and western religion, science and technology. He further explained that often data on IKS are distorted to confirm the hypothesis of non-Africanist scholars" (p. 131). Also [21] stated that younger generations who are exposed to formal education are less interested in indigenous knowledge, viewing such knowledge as old-fashioned and primitive. IK is at risk of becoming extinct if appropriate measures are not taken to manage it to assure its accessibility to future generations [16].

\section{Role of Technology in preserving Indigenous Knowledge (IK)}

Because technology is the center of human activities, there is need to introduce it to complement the traditional methods of IK preservation which revolves around "oral tradition" and is currently facing numerous challenges and leading to this traditional wisdom becoming extinct. However, the introduction of technology must be minimalistic [10] but geared towards user-friendliness, more context sensitive and representative of the local communities. Doubtlessly ICT, in general has enormous potentials in improving the availability, accessibility and dissemination of IK while adding value to the IK system. In previous study [25], it is indicated that ICTs play major roles in improving the availability of indigenous knowledge systems and enhancing its blending with the modern scientific and technical knowledge. This is possible through the use of, for example information systems designed specifically for this purpose.

Using Information Systems to preserve IK is inevitable because ICTs has become the platform for all human activities, including transmission and reliable information access from anywhere and at any time. ICTs such as computers, Internet, and other mobile devices have been used by service providers for service delivery to beneficiaries, project task collaboration and used in real time communication and exchange of information. From this viewpoint, using Information Systems to preserve IK is central to improving the transmission and exchange of IK between the remote local communities and modern societies where western knowledge is practiced. An earlier report [10] highlighted that, the application of ICT is essential to stimulate the flow of IK and incorporation of modern scientific and technological understanding of traditional knowledge. The main use of ICTs in preserving IK could be to a) capture, store, transmit and disseminate IK to preserve traditional wisdom for future generations b) promote ease of accessibility of IK c) promote ease of integration of various forms of 
knowledge of heritage d) promote easy integration of formal and non-formal body of knowledge into the education programs e) potential promotion of copyright and patent on local knowledge, and f) promotion of economic value added to local knowledge. All these benefits of information systems can significantly improve access and availability of IK to especially the younger generation who are more technology compliant than the older generations.

\section{METHODOLOGY}

This study is a work-in-progress with a goal to probe how Information Systems can be used to preserve and disseminate IK, in an era of technological explosion. A descriptive and qualitative method was used at this stage of the study. The qualitative approach was used in the focused group interviews with both the students and staff members. However, in later stages, quantitative methods will be employed in the form of questionnaires, whereby the outcome and the issues that arose from the focused group discussions will be used to formulate the questionnaires to be administered to respondents. The target sample of the study was 90 students and 15 lecturers. The sample was selected using stratified random sampling technique from this higher institution of learning. A qualitative approach was used to prepare the two group discussions which were conducted to discuss the issues surrounding IK from three broad themes; a) Do you think that IK is important in the sustainable development, and why? and b) what is the main challenges surrounding IK as opposed to Western knowledge? And c) Do you think that Information System is essential for the preservation of IK of the local communities? How? Following this discussions, a questionnaire that is divided into five sections will be designed and administered to respondents and analyzed to verify the issues which came up during the discussions. The first part will contain the background of the respondents, the second part will comprise of direct questions requiring yes/no and multiple-choice items, the third part will comprise of a Five Point Likert-scale type of questions. The fourth part will contain the questions regarding opinions of the respondents on examining the use of Information Systems to preserve Indigenous Knowledge. Lastly, the fifth part will comprise of sections where respondents will be free to give their recommendations on the use Information Systems to enhance preservation and dissemination of IK.

\section{ANALYSIS OF RESULTS}

From the discussions, it is generally agreed that IK is important for community development although the discussants seemed more concerned with cultural and historical preservation, creative in skills enhancement aspects of IK than the actual community development aspects. This meant that, the discussants valued the local skills related to activities that leads development than the real development process as a whole. Also, the participants seemed more concerned about the historical events and cultural practices performed by the locals such as the rituals and other celebrations than how these contributes to the development process. To some extent, the discussants may not have been aware of the context of development in question. However, on the issues of socio-economic empowerment through commercialization of culture, management, and control of resources shows that they value the role played by IK in improving lives of local community members. This was highlighted in discussion topics such as commercialization of cultural performances, like traditional dances, cultural tourism like visiting some cultural sites for a fee, and commercialization and value addition and branding of herbal medicine. This topic drew more interests from the discussants and several examples were also cited.

On the second theme, the discussants were well aware of the challenges facing IK especially in the Ugandan context. This could have been due to the fact that, much as the discussants were from a varying tribal and cultural backgrounds, they cited these challenges from the viewpoint of cultural inclination since it was the main reason for pointing out that IK is an important component of development. The discussants also seemed to realize the weakness of oral tradition as the sole traditional method for transmitting and preserving IK and they also seemed to understand the importance of documentation as a way to preserve IK for future reference. Lack of documentation results in another problem of fear IK becoming extinct since its holders die with their knowledge. This is cited with the example of herbalists becoming fewer in number with time thereby leading to lose of knowledge about herbal medicines. Another challenge is that IK is ignored by central government in the development process. The discussants could have cited this because they have a clear understanding of governance in that, solutions to community problems should come from the grassroots. And they also cited that most government policy formulation is a top down approach. And most government interventions to community problems are not very effective because it ignores the grass-roots approach and by ignoring such approach, they ignore with it some very valuable IK which could have been effective in addressing contextual challenges because there is no one-size-fits all approach to national problems. Also, another challenge of IK was cited as being gender insensitive because it highly favors men. This is because most cultural practices disregards women and treats them as a weaker gender, and without rights neither to own properties nor to inherit. Most cultures bars women from decision making since men are the heads of the families and therefore heads of communities in which they live. Here it clearly shows that the discussants were able to relate IK and cultural practices that inhibit development since gender equality is advocated for worldwide and it's known to be a recipe for development in all contexts. It is clear that moving from rural areas and settling in urban areas, for reasons of 
access to quality education and sources of employment brings challenges to IK in the sense that it creates a gap between the holders of IK who are elderly and less formally educated people in remote areas and the seekers of IK who are the younger generation who live and work in urban centers. Lastly, the discussants argued that there is also a negative attitude of most of the young people especially the elite class and those who attend formal education since they think that IK is primitive in nature and anything Western is modern and civilized. They do not pay attention to the fact that, this knowledge they consider primitive has effectively sustained these communities for decades and centuries.

The discussants agreed wholesomely that using Information Systems specifically to gather, record, store and disseminate IK in digital format would help to protect it from becoming extinct because this is vital knowledge which is hard to get since the holders and the seekers are separated by a gap and unsuitable method of transmission, the oral tradition. This Information System would, therefore, act as a gap to access IK since technology is now inevitable in all aspect of human life, and especially for the dot com generation as well as the social media generation.

\section{FINDINGS}

These findings are from the two separate discussions held on the three themes. The first discussion was conducted with the staff (teaching and library) and the second, with the students. In both the discussions, similar answers were identified across all the themes. The findings of this study are organized into three sections in order to provide answers to the research questions as analyzed below:

A. Research Question 1: Do you think that IK is important in the sustainable development, and why?

This first single choice question of the survey is to determine if IK is important in the sustainable development based on students and staff members' opinion or experience and why. 28 out of 29 participants agreed that is important in the process of development and the reasons varied greatly, but cultural preservation, creativity in skill enhancement through craftsmanship and other creative methods such as farming, fishing, and house construction, keeping track of history and selfdiscovery topped the discussions, in that order. Other reasons included socio-economic empowerment through commercialization of cultural tourism, socialization, management and control of communities and their resources, and alternative sources of other knowledge, for example, predicting rain by observing the wind direction and patterns as well as cloud formation and movements. Also, participants cited reasons such as IK is the foundation of in-depth understanding of local problems, a way to impart a broader sense of responsibilities, acceptable norms and ethical standards to the younger generation to prepare them for future adult life since the local societies have a way of teaching gender roles to the younger people to prepare them to become responsible and hardworking and self-reliant adults. The only one participant who said that IK is not important for development, cited that it is time and energy consuming since all activities are manual. For example, the traditional farming methods is so energy draining since small tools such as hand-made hoes are used to dig with human energy as opposed to the modern mechanized faming methods that uses less man power but more intelligence and can be done on a large scale.

\section{B. Research Question 2: What are the main challenges surrounding IK as opposed to Western knowledge}

The findings from this research question identified several issues as the main challenges. The issues which topped the list of the discussion was inadequate documentation of IK, since oral tradition is the sole preservation and transmission methods used in almost all local settings. It was also noted that IK is ignored by the central government system, because all government interventions and policies does not include any considerations of contextual IK to address local, and regional challenges. It was also pointed out strongly that, IK is excluded from the formal education system, whereby the entire education system portrayed western imposed systems that ignores several contextual and environmental factors. Due to lack of documentation, oral tradition is unreliable method of preservation is a challenge because the death of knowledgeable elders leads to loss of critical IK. The others included issues surrounding lack of enough information on IK, gender insensitive cultures since it favors only men, negative attitudes towards cultural practices especially in these days of formal education, modernization and urbanization. The inflexible cultures that fail to adapt with changing times and needs. They go on to explain that IK is overshadowed by Western knowledge, some of the practices especially in agriculture degrade the environment, and lastly, the discussants stressed that some of the IK practices create division within communities since its basically unique to tribes confined within certain locality and regions. This means that, some of the IK practices are non-unifying factors or does not contribute to national development and national unity.

\section{Research Question 3: Do you think that Information Systems is essential for the preservation of IK of the local communities? How}

The findings from this research question also indicate that 28 out of 29 discussants agreed that Information Systems is essential in preserving IK because of a large number of people especially the youth, use technology daily and for nearly anything. A narrow variation in the responses was encountered. Most of the responses revolved around using Information Systems for digitizing IK and keeping records and to guarantee availability provide easy access, management, and dissemination of the contents from anywhere. It was, however, unclear why one of the discussants said that Information System is not important in preserving IK since there was no 
explanation that reasoning. Probably the question may not have been clear to that particular participant.

\section{DISCUSSION}

The perceptions of students and staff members remain very important in the use of information systems to preserve IK. Thus, the primary purpose of this study is to examine the use of information systems to preserve IK based on students' and staff members' perceptions. This is because of the role of the university in the transformation and development of communities through in-depth research. In particular, we are very interested to study how Muni University students and staff members think about preservation of IK using information system and if the technology can help preserve IK.

The finding from research question one (1) shows that it is clear that local community members fairly understand the importance of IK and are aware that it is fading and that there is the need for interventions to protect it from becoming extinct. This outcome is consistent with the study conducted by other researchers [16] where it was also noted that little IK has actually been captured and recorded. Therefore, there is an urgent need to manage IK to enhance its availability for developmental initiatives before much of it is completely lost. It is also supported by [26] where it was noted that IK must be managed because it plays a crucial role in the sustainable development of society. It is, therefore, appropriate to conclude that there is need to preserve and protect Indigenous Knowledge. Our idea is therefore to use information systems to adequately record, maintain and preserve IK for the future generation who no longer fit with oral tradition due to changes in life styles, modernization and globalization.

Taking the challenges facing IK into account, the findings from research question indicate that inadequate documentation, coupled with IK by ignored by the central government system and policies, and it being excluded from the formal education system, as well as the death of knowledgeable elders who die with their knowledge, and the lack of enough information on IK, including the gender insensitive cultures that favors only men, plus the negative attitudes towards cultural practices surrounding the IK especially in these current days of modernization and urbanization, and western knowledge are some of the challenges facing Indigenous Knowledge. These findings are also reported in other earlier studies conducted by [5], [13], [14], [12], [16], [17], [19], [20]. [21]. It is also consistent with [15] who identified political pressures, cultural integrity, social and economic pressures, territorial pressures, exploitation of traditional knowledge, development policy, and globalization and trade liberalization as threats to Indigenous traditional knowledge.

It is clear from the result of the research question three (3) that technology offers significant potentials in the preservation and dissemination of IK but technology should be used in ways to protect the origin and ownership of the traditional knowledge because it is unique to a local community. This outcome is consistent with the study conducted by [21] where Tanzania Development Gateway database on IK provides a platform where IK is captured, stored and disseminated; and also integrate it with modern science and technology to enhance information dissemination; They clearly understand the importance of IK and how it could change many lives and be used to find solutions to contextual societal problems and challenges. This outcome is consistent with the study conducted by [27] who observed that $80 \%$ of the world's population depend on IK to meet their medicinal needs and at at least $50 \%$ rely on IK for food supply. It is also supported by [21], [13], [28], [29] who argue that IK is recognized as having relevance to the daily life of most individuals, economic development, cultural preservation and political transformation, which lead to poverty reduction; rely on it for medicinal and herbal needs, food supply, conflict resolution and spiritual growth, to resolve potential conflicts arising from competing claims to land. IK is an underutilized resource in the development agenda, particularly in developing countries. It is a tool to be employed by all development partners in order to achieve effective solutions to community problems.

\section{CONCLUSION \& RECOMMENDATIONS}

Based on the issues raised and discussed in this paper and those in related literature, we conclude the following; first, IK is an essential resource for sustainable community development but is either underutilized, neglected or ignored for various reasons such as those that are associated with western knowledge, lack of framework for IK inclusion, and formal education and as a result of modernization and globalization. Because of its ineffective oral tradition methods of preservation and transmission, IK is fading off and it is feared that it will become extinct. Second, technology presents significant potential in preserving IK since the traditional preservation method of oral tradition is no longer effective. Therefore, digital preservation shall add value to IK and as such, we expect that using Information Systems to preserve and transmit IK would increase its relevance and therefore increase the need to search for and integrate it into the relevant areas of development by stakeholders, particularly to education, agriculture and health since demand for herbal medicine is increasing. Using Information Systems shall also enable preservation of the collective memory of local communities by collecting, storing, archiving and disseminating historical and cultural heritage of unique communities in a central repository for access from anywhere and at any time, therefore acting as a bridge to the IK gap among communities. Third, digitizing IK has a potential for commercialization of cultural tourism due to the documentation of historical and cultural heritage which increases value to such cultures. Lastly, the preservation of IK will also lead to bridging the mindset gap of the new generation of community members to believe in their heritage and knowledge as opposed to the most 
developing country mentality of thinking that any knowledge generated by the West is superior to their own IK. However, significant challenges still remain and need to be overcome to ensure that such studies realize their goals both to the owners and users of the knowledge. Although they may be technical challenges, there is also socio-economic, political and inadequate knowledge of local knowledge.

From the conclusions above, we do recommend that there should be massive sensitization about IK and its relevance in solving human problems as well as its value in education. Therefore, educational institutions ought to adopt IK into their learning environment to facilitate dissemination and awareness creation about its value. Secondly, all development stakeholders particularly governments and non-governmental organizations should exhaust all local options in formulating solutions to community problems and should step up its efforts to integrate IK into development plans and objectives. Due consideration should be given to IK and a framework for addressing origin and ownership of IK should be formulated. IK center(s) should be established to enlighten the masses about IK and to provide reliable access to such vital local knowledge. Thirdly, we recommend further research about the contextual use of technology to preserve and disseminate IK due to the fact that has changed the way we search for information and knowledge especially at the time traditional the methods of preserving and transmitting IK is no longer sustainable. Lastly, research needs to be conducted about the use of other media such as TVs, social media, and broadcast radio to transmit and disseminate IK because these new media has changed the way people socialize, relate and exchange knowledge.

Since this is a work-in-progress, it marks the beginning of a longitudinal and ethnographic research into how to improve the preservation and dissemination of IK especially to the urban dwellers and the educated population who have no direct or consistent connection to the remote areas which is the cradle of IK.

\section{ACKNOWLEDGMENT}

This research was a result of the collection of many people who have contributed directly or indirectly. So our appreciations are owned by so many people who contributed to the completion of this research. First, we would like to express our gratitude to Muni University for the excellent facilities provided to complete this research. Finally, the authors extend their appreciation to the staff of Muni University for their valuable advice and comments. Lastly, a special appreciation to $\mathrm{Mr}$. Nkamwesiga Lawrence for final proof reading and doing spell check on the final document for submission for publication.

\section{REFERENCES}

[1] V. T. Itai and M. Chikwanha, "The Role of Indigenous Knowledge Systems in the Management of Forest Resources in Mugabe Area, Masvingo, Zimbabwe,"
Journal of Sustainable Development in Africa, vol. 13, no. 3, p. 18, 2011.

[2] IIRR, "Recording and using indigenous knowledge: A manual," IIRR, Silang, 1996.

[3] M. Mawere and S. A. Nyamekye, Between Rhetoric and Reality: The State and Use of Indigenous Knowledge in Post-Colonial Africa, Engelska, 2015, pp. 220.

[4] J. K. Sarkhel, "Strategies of Indigenous Knowledge Management in Libraries," Qualitative and Quantitative Methods in Libraries (QQML), vol. 5. pp. 427-439, 2016.

[5] L. T. Tjiek, "Desa Informasi: The role of digital libraries in the preservation and dissemination of indigenous knowledge," The International Information \& Library Review, vol. 38, no. 3, pp. 123-131, September 2006.

[6] N A. Akinwale, "Digitisation of Indigenous Knowledge for Natural Resources Management in Africa," in Proceedings of the 2012 Summit of the African Educational Research Network, 18-20th May, 17 North Carolina State University \& DoubleTree Hilton, Raleigh Brownstone University Hotel, 2012.

[7] J. Hunter, "The Role of Information Technologies in Indigenous Knowledge Management," Australian Indigenous Knowledge and Libraries Journal, vol. 36, no. 2, pp. 109-124, 2005.

[8] "Indigenous Knowledge For Development A Framework For Action," Knowledge and Learning Center Africa Region World Bank, 1998.

[9] V. K. Chouhan, "Protection of Traditional Knowledge in India by Patent: Legal Aspect," IOSR Journal of Humanities and Social Science (JHSS), vol. 3, no. 1, p. 8, 2012.

[10] S. Lodhi and P. Mikulecky, "Management of Indigenous Knowledge for Developing Countries," Communication and Management in Technological Innovation and Academic Globalization, p. 5, 30 October 2010.

[11] K. Boven and J. Morohashi, "Best Practices using Indigenous Knowledge," Nuffic, The Hague, The Netherlands, and UNESCO/MOST, Paris, France, Hague, Paris, 2002.

[12] S. Amanda, "A Different Way of Knowing: Tools and Strategies for Managing Indigenous Knowledge," International Journal of Libraries and Information Studies, 19 May 2008.

[13] J. Mapara, "Indigenous Knowledge Systems in Zimbabwe: Juxtaposing Postcolonial Theory," J. Pan African Stud., vol. 3, no. 1, pp. 139-155, 2009.

[14] R. Ulluwishewa," Indigenous knowledge, national resource centers and sustainable development," Indigenous Knowledge and Development Monitor, vol. 1, no. 3. pp. 11-13, 1993.

[15] G Kelly (AIATSIS), Report on Threats to the Practice and Transmission of Traditional Knowledge Regional Report: Asia and Australia, Phase II of the Composite Report on the Status and Trends Regarding the Knowledge, Innovation and Practices of Indigenous Peoples and Local Communities relevant to the Conservation and Sustainable Use of Biodiversity, 2005, UN Doc: UNEP/CBDWG8J/4/INF/4, p 25.

[16] E. T. Lwoga, P. Ngulube, and C. Stilwell, "Managing indigenous knowledge for sustainable agricultural development in developing countries: Knowledge management approaches in the social context," International Information \& Library Review, vol. 42, no. 3. pp. 174-185, 2010.

[17] E. T. Lwoga and P. Ngulube, "Managing indigenous and exogenous knowledge through information and communication technologies for agricultural development 
and achievement of the UN Millennium Development Goals," Libraries and information services towards the attainment of the UN Millennium Development Goals, Berlin, pp. 73-88, 2008.

[18] S. L. Whatman and P. Duncan, "Community participation in indigenous education: Learning from the past, in policy and practice", Introductory Indigenous studies in education: The importance of knowing, pp. 117-138, 2005.

[19] C. T. Eyong, "Indigenous knowledge and sustainable development in Africa: a Case study on Central Africa," Indigenous knowledge systems and sustainable development: Relevance for Africa, pp.121-139, 2007

[20] L. M. Semali and J. L. Kincheloe, "What is indigenous knowledge? Voices from the academy," New York, NY: Falmer Press, 1999

[21] J. Msuya, "Challenges and opportunities in the protection and preservation of Indigenous Knowledge in Africa," International Review of Information Ethics (IRIE), vol. 7, no. 9, p. 8, 2007.

[22] J. Smylie, C. M. Martin, N. Kaplan-Myrth, L. Steele, C. Tait and W. Hogg, "Knowledge translation and indigenous knowledge," Circumpolar Health 2003, pp. 139-143, 2003.

[23] R. Ataur, "Development of an Integrated Traditional and Scientific Knowledge Base," United Nations, Geneva, 2000.

[24] M. H. W. J, "The Influence of Information Behaviour on Information Sharing across Cultural Boundaries in Development Contexts," Information Research: An International Electronic Journal, vol. 14, no. 1, p. 13, March 2009.

[25] A. Lishan, "Information and Communication Technologies, Knowledge Management and Indigenous Knowledge: Implications to Livelihood of Communities in Ethiopia," 1991.

[26] P. Ngulube, "Managing and Preserving Indigenous Knowledge in the Knowledge Management Era: challenges and opportunities for information professionals Information Development," vol. 18, no. February 2016.

[27] J. B. Nyumba, "The Role Of The Library In Promoting The Application Of Indigenous Knowledge (IK) In Development Projects," in World Library and Information Congress: 72nd IFLA General Conference and Council, Seoul, Korea, 2006.

[28] N. Gorjestani, "Indigenous Knowledge for Development," pp. $1-8,2000$.

[29] A. Steiner, "Indigenous knowledge in disaster management in Africa," International Council for Science, 2008

\section{Authors' Profiles}

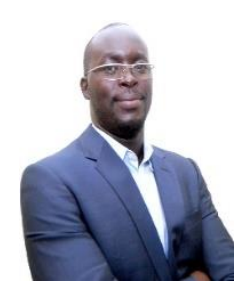

Josephat O. Oroma is a Ph.D. student at the University of Eastern Finland, Joensuu Finland and Lecture at, and the Acting Head of Department of Computer and Information Science, Muni University, Uganda. His research interests include Innovative and effective teaching methods, Gender and IT Education, ICT4D, Educational Research, and Cognitive Science. And more recently, he is gaining interests in Artificial Intelligence and Data Science.

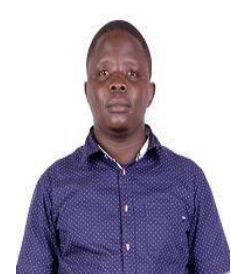

Guma Ali is an Assistant Lecturer at the Department of Computer and Information Science, Muni University, Uganda. His research interests are in the fields of Educational Technology, Mobile Learning, IoT, Cloud Computing, and ICT for Sustainable Development.

How to cite this paper: Josephat O. Oroma, Guma Ali, "Examining the Use of Information Systems to Preserve Indigenous Knowledge in Uganda: A Case from Muni University", International Journal of Modern Education and Computer Science(IJMECS), Vol.10, No.5, pp. 36-43, 2018.DOI: 10.5815/ijmecs.2018.05.05 